Session 2793

\title{
Multi-Media Fluid Mechanics
}

\author{
G. M. Homsy \\ Stanford University \\ (Current affiliation: University of California at Santa Barbara)
}

\begin{abstract}
This paper summarizes the results of a two-year project sponsored by the National Science Foundation (NSF Project "Hypermedia Fluid Mechanics: Teaching Modules for the Next Century", NSF/DUE 9752199). This project was a multi-investigator effort to develop a CDROM, compatible with a wide range of platforms, processors, and operating systems, containing multimedia materials for teaching undergraduate fluid mechanics. It was released in Fall, 2000, and is being marketed by Cambridge University Press under the title "Multi-Media Fluid Mechanics" $(M M F M)$. Its co-authors are: G.M. Homsy, H. Aref, K.S. Breuer, S. Hochgreb, J.R. Koseff, B.R. Munson, K.G. Powell, C.R. Robertson, and S.T. Thoroddsen. A short demonstration of $M M F M$ may be viewed on the Cambridge University website: http://www.cup.org/books/homsy/default.htm This paper gives a summary of the background and objectives of the project and some of the features and capabilities of MMFM.

I. Introduction and Educational Challenges

Given its central role in science and technology, it is not surprising that fluid mechanics remains a core foundational subject within many disciplines of engineering and applied science, including Mechanical, Civil, Environmental, Petroleum, Aeronautical, and Chemical Engineering, Meteorology, Oceanography, Applied Physics, Applied Mathematics, Engineering Mechanics, and Bioengineering. As the demand for understanding of transport processes increases, fluid mechanics is being required for students in materials and semiconductor processing, thus involving students from Materials Science and Electrical Engineering. It is also true that many graduate students in Earth and Planetary Sciences, Geophysics, and Applied and Condensed Matter Physics take an advanced undergraduate courses in fluid mechanics as important background to their thesis research. It is clear that by its nature, fluid mechanics is both an interdisciplinary and interprofessional subject, constituting one of the most general and important subjects in the undergraduate curriculum. Not only is it a fundamental subject, but it also provides essential physical understanding and methodology in many emerging fields and advanced technologies such as advanced transport systems, the processing of novel and exotic materials, semiconductor processing, global environmental modeling and protection, improved energy extraction and use, processing of chemicals into new products, atmospheric processes, improved artificial organs and devices for the treatment of disease, and many others. Knowledge of the principles of fluid mechanics and the ability to solve fluid mechanics problems are a critical component of the practice of engineering at the BS level.
\end{abstract}

Proceedings of the 2001 American Society for Engineering Education Annual Conference and Exposition Copyright @ 2001 , American Society for Engineering Education 
Fluid mechanics is typically taught in the junior or senior year as a required engineering course. The fundamental basis of the subject is reasonably easy to state, since it is firmly rooted in the principles of conservation of mass and Newton's Second Law of Motion. The mathematical statement and the consequences of these principles for continuously deforming media are, however, often confusing and daunting to the student. The subject is mathematically sophisticated, involving (i) time dependent, 3D partial differential equations, (ii) the essential use of vectors, tensors, and vector analysis, (iii) an often bewildering array of dimensionless groups, (iv) the dependence of fluid phenomena on the various parameters over a wide range of those parameters, and (v) the surprising behavior of fluids that often runs counter to a naive set of intuitive concepts.

Conquering abstract concepts in this important field has positive consequences in other parts of the undergraduate engineering curriculum. Fluid mechanics is one of the earliest field theories in the physical sciences, and as such provides the student his or her first exposure to a continuum description of mass, momentum, energy, force, and other quantities in a familiar, mechanical context. These concepts will emerge often in later studies, where they may be set in considerably more abstract contexts, e.g., in electromagnetic field theory. Historically, the concepts of fluid flow have always played an important role in building intuition about general field descriptions in the physical sciences and represent the first serious application of vector analysis to engineering science. Educational modules based on modern interactive multimedia tools that help students learn and retain knowledge of field theories and that help conceptual thinking will have important additional benefits in other mathematically oriented and abstract parts of the undergraduate curriculum and in other domains of engineering theory and practice.

Based on many decades of teaching undergraduate fluid mechanics, we identified the following challenges to be met by the next generation of educational materials in fluid mechanics.

\section{Connect concrete physical phenomena with mathematical / analytical abstraction.}

Many of the important concepts in fluid mechanics, e.g. vorticity, velocity, and pressure fields, and vector analysis are not particularly tangible to beginning students. Both new and experienced teachers point to this as one of the major challenges in education: to teach how physical concepts are expressed in mathematical terms, and to develop the ability to move freely from the mathematical context to physical reality and back, building up understanding and intuition in the process. Both experiment and theory are useful in making this connection.

\section{Incorporate modern computer simulation into the curriculum.}

While scientific computing has achieved equal standing with experimentation and analytical theory in research, it has not yet had a significant impact on the teaching of fluid mechanics. This is a serious challenge that must be addressed. Learning is enhanced by the ability to simulate and visualize flows which cannot be expressed by analytical theory. The use of computation is invaluable in providing the ability to systematically vary parameters and study the evolution of flow structures and flow physics that occurs as a result. Parametric dependence, including geometric dependence, is one of the most difficult concepts to teach, and the efficacy of interactive, computational based modules cannot be overemphasized. However, the time Proceedings of the 2001 American Society for Engineering Education Annual Conference and Exposition Copyright $\odot$ 2001, American Society for Engineering Education 
involved in writing and validating of robust software for flow simulations often puts the use of computer simulation outside the range of student projects on the one hand, and the time and expertise of the instructor on the other. Delivery of such robust software through multimedia interactive modules will result in enhanced capabilities that can be widely disseminated.

Expose students to state-of-the-art experiments in fluid mechanics.

Using sophisticated experimentation to elucidate physical phenomena was a key motivation for our project. Experiments and the qualitative and quantitative knowledge they express are invaluable in reinforcing concepts from the mathematical theory of fluid flow. Modern flow visualization using high-speed cameras, along with tracers such as small particles, bubbles, and laser induced fluorescent dyes, has greatly extended the range of flow visualization techniques and the insight gained from them. Such images are indispensable in building physical intuition. However, construction of state of the art apparatus is expensive, difficult to maintain, and often beyond the financial resources of many colleges and universities, rendering them inaccessible for student use in beginning classes, and at many universities they may not even be available on campus. $M M F M$ brings such experiments into the classroom using modern technology.

Increase student learning by generating curiosity and exploiting the medium.

Undergraduate instruction in fluid mechanics suffers from the false perception that the subject is "mature", and that nothing new has happened in it for a long time. The usual "talk-and-chalk" format of most lecture courses reinforces this perception. A course devoid of visual aids and computer technology comes across as decidedly "low tech" to today's students. Interactive computer exercises, visualizations, and demonstrations can have considerable impact in generating interest and excitement in this vital subject among the best and brightest students, yet they are hardly used in undergraduate education in fluid mechanics today. These can be developed to the fullest and exploited in many different ways as an aid to enhanced learning.

We believe that interactive multimedia modules that emphasize experiments and computations can be extremely effective in meeting these challenges. Our project addresses the issues of access to experimental and computational knowledge and capabilities in a vigorous, modern way.

\section{Description}

MMFM contains four modules covering the subjects of Dynamics, Kinematics, Boundary Layers, and History. Each has a number of topics, and each of these topics is developed in a number of pages. As a result, there are several dozens of topics, well over 250 videos in Quicktime format, and approximately 100,000 words of descriptive text. There is a high level of interactive material, including simulations, virtual labs, and video visualizations, animations, and demonstrations of fluid phenomena material that are intended to complement any of the standard undergraduate fluid mechanics textbooks. These visual materials are in 1/4 screen video format, accompanied by explanatory text, and are viewed using standard QuickTime software. All the visual material can be accessed through a separate Video Library, thus enhancing its use by instructors in the classroom. Navigation between and within the modules is clear and easy, and 
is facilitated through the use of hyperlinks. Of particular note are the following special features and capabilities.

\section{Simulators.}

Students use computer simulations to vary parameters and/or illustrate a principle. $M M F M$ has three such simulators: Molecular Dynamics, Integration of the Laminar Boundary Layer Equations, and the Potential Flow Builder.

\section{Virtual Labs.}

In these virtual labs, students take quantitative data from videos and/or use a simulator to compare theory and experiment. They include matching potential flow theory with Hele-Shaw experiments; verifying dynamic similarity in vortex shedding; measurement of laminar boundary layer growth in the Blasius boundary layer; and verification of self-similarity in the impulsive start of a plate.

\section{Charts and Demonstrations.}

This extensive series of over 50 charts and demonstrations includes side-by-side comparison of flows at different Reynolds numbers and for different shapes; comparison of potential flow theory with bluff and streamlined shapes; flow structure around sports balls; origin of drag forces as a function of Reynolds number and body shape; techniques and methods of flow visualization; and many others.

The Flow Gallery.

In this gallery, the student may view any of a large number of videos of fluid phenomena, including flight, vortices, mixing, swimming, thermal plumes, airplanes, natural and environmental flows, etc. These are arranged pairwise according to the Reynolds number, and following a description of the principles involved, a short illustrative video of each may be viewed.

The presentation of this paper at the ASEE Meeting will consist of a short demonstration video, followed by having the full version of $M M F M$ available for testing and use by interested parties.

Marketing details for MMFM may be viewed by going to the CUP website (www.cup.org) and searching on the ISBN \# 0-521-78748-3 or on Author = Homsy. Alternatively, a short demonstration of $M M F M$ may be viewed at the following url: http://www.cup.org/books/homsy/default.htm

One of the most attractive features of $M M F M$ is its low cost; $\$ 20 /$ copy. The concept and our hope is that by pricing it low, students will be encouraged or required to purchase their own copy for personal use.

\section{Organizational Details}

The authors, G.M. Homsy, H. Aref, K.S. Breuer, S. Hochgreb, J.R. Koseff, B.R. Munson, K.G. Powell, C.R. Robertson, and S.T. Thoroddsen, were organized into a set of working groups with 
informal responsibility for each of the four modules. The content and rough format were first outlined, followed by extensive critiques by the entire group of authors before text and visuals were assembled. Specifications of video material and animations were determined and sources identified. Story boards were then assembled and the text produced. All text was then read by a second (and in some cases, a third) member of the working group to ensure clarity and precision.

Key issues in the success of any teaching/learning aids are quality of the learning experience, accessibility, user-friendliness, and cost. Foremost emphasis was placed on the quality of the modules, in order to ensure that they offer intellectual stimulation, clear presentation, high visual quality, and a wealth of examples. Ease of access via CD-ROM was addressed by designing the modules to be as platform independent as technically feasible, and the navigation to be clear and easy to use. We engaged the services of a professional multimedia production company, Pacific Media, Santa Clara, CA, whose prime responsibilities were the production of the navigation tools and the underlying programming to fully integrate the simulators into the software environment. This turned out to be a very well-considered decision. Commercial companies afford access to state of the art digitization, data compression, graphic artists for animations and graphics, and high-level programmers, which is what gives $M M F M$ its highly polished and professional look and feel. In addition, Pacific Media established creative web-based tools by which the working group members could work remotely on the modules. These tools included (i) maintaining an html version of $M M F M$ that ran in a browser environment, accessible through a web-site maintained by them, (ii) establishing a Video Library that contained all the current videos, which authors could view remotely in order to select suitable material for inclusion in their particular module, and (iii) tracking of changes and documentation in the text of the modules.

As required by any NSF project of this magnitude, we were assisted by a National Visiting Committee whose membership included J. Gollub, L. Smits, J. Riley, and P. Hancock. In their reports to the NSF/DUE, the NVC stressed the importance of continuity so that the initial substantial effort involved in getting $M M F M$ established not be lost due to lack of continuous funding. At the time of writing (Winter, 2001), we are working with the Division of Undergraduate Education of the NSF to launch a follow-on project. Our vision is to complete a suite of modules covering a wide range of fluid phenomena over the next decade

An extensive set of formative evaluations and testing was conducted for $M M F M$, which included two rounds of testing of the content, concepts, and 'look and feel' of the navigation. These were conducted by MA students in the School of Education from Stanford University, and comprised part of their project requirements for the degree program in professional evaluation. The evaluations themselves involved undergraduate students, graduate teaching assistants, and professors of fluid mechanics at Stanford, San Jose State, and Santa Clara Universities. Final summative evaluation of $M M F M$ are underway at the time of writing, and will comprise an important part of our final report to NSF. The feedback we have received so far from our formative evaluations, the early summative evaluations from Fall, 2000, and informal feedback from the community, has been extremely positive. $M M F M$ was chosen by the NSF/DUE staff to be featured in the NSF Showcase at the ASEE Meeting in St. Louis, MO in June 2000. We are confident that we have created a robust framework for innovative curricular multimedia resources for teaching and learning fluid mechanics for the 21 st century. We are also hopeful 
that some of the features and experience from $M M F M$ will provide effective models for the promulgation of multimedia-based projects into other areas of the undergraduate curriculum.

\section{GEORGE M. HOMSY}

George M. Homsy is a Professor of Mechanical and Environmental Engineering at the University of California at Santa Barbara. He was previously on the faculty of Chemical Engineering, Stanford University, from 1970-2000. He received all his degrees in Chemical Engineering, including his BS from the University of California, Berkeley, and $\mathrm{MS} / \mathrm{PhD}$ degrees from the University of Illinois, Urbana-Champaign. He served as the Principal Investigator for "Multi-Media Fluid Mechanics". His teaching and research interests are in fluid mechanics, thermal transport, hydrodynamic stability, and applied mathematics. He is a member of the AIChE, ASEE, and a Fellow of the APS. 